\title{
Guest editorial: Distributed control and optimization of wireless networks
}

\author{
Yongmin Zhang ${ }^{1}$ - Wenchao Meng ${ }^{2} \cdot$ Heng Zhang ${ }^{3} \cdot$ Preetha Thulasiraman $^{4}$. \\ Tom H. Luan 5
}

Published online: 7 January 2017

(C) Springer Science+Business Media New York 2017

\section{Introduction}

Wireless networks, as compared to its wired counterpart, bring improved coverage yet enhanced convenience and low cost in deployment. As a result, a couple of emerging wireless networks, such as cognitive radio networks, wireless sensor networks, WiMAX/LTE, adaptive communications, etc., have proliferated to meet the surge wireless traffic demand and provide ubiquitous communication at diverse application scenarios.

Heng Zhang

ezhangheng@gmail.com

Yongmin Zhang

ymzhang@zju.edu.cn

Wenchao Meng

wenchaomeng@doe.carleton.ca

Preetha Thulasiraman

pthulas1@nps.edu

Tom H. Luan

tom.luan@deakin.edu.au

1 Zhejiang University, Hangzhou, China

2 Carleton University, Ottawa, ON, Canada

3 Huaihai Institute of Technology, Lianyungang, China

4 Naval Postgraduate School, Monterey, CA USA

5 Deakin University, Geelong, Australia
While the emerging wireless technologies have greatly promoted the availability and applicability of wireless services, they are still far from ideal due to the intrinsic flaws of wireless channels, such as limited and dynamic data transmission capacity, rampant channel interferences, constrained coverage range, scarce spectrum resource, and vulnerability, etc. There thus exist pressing need to develop more efficient wireless data transmission solutions with the optimal control and adaptation of the communication system on the aspects of on resource allocation, security and privacy protection, power control, spectrum management, distributed control, parallel computing and networked control.

As wireless technologies not only pervade the everyday lives of people, but is also widely applied in the industrial manufacturing and process, it is necessary to optimally and intelligently adapt the wireless solutions to meet the different industry service requirements of specific applications on throughput, communication delay, robustness and reliability, etc. This special issue will focus on broadly in the area of network optimization and control that can be incorporated in the functionality of a wireless network in both residential and industrial scenarios, and will try to identify new opportunities and research directions in the emerging wireless technologies.

This special issue will focus on network optimization and distributed control schemes that can be incorporated in the functionality of a wireless network in both residential and industrial scenarios, and will try to identify new research issues, opportunities and directions in the emerging wireless technologies. We have received more than 60 submissions, and each submission had been rigorously reviewed by at least three experts, and finally we selected 29 papers for publication. 


\section{The issue at a glance}

The special issue consists of 29 papers in several research areas. For the convenience of the reader, we classify the accepted papers into the following four categories: data collection optimization, performance optimization, protocol design and applications.

\subsection{Data collection optimization}

Data collection is an important aspect of any type of research study in Wireless Networks. How to design a efficient data transmission scheme to optimize the data collection performance has been addressed in the special issue.

In static wireless network 1) The paper by Ruilong Deng et al. studied the network utility maximization (NUM) problem in dynamic-routing rechargeable sensor networks (RSNs), and proposed a joint rate, routing and energy management to obtain the optimal data collection; 2) The paper by Yanjun $\mathrm{Li}$ et al. proposed a dynamic frame length and charging time adaptation scheme (DFCA) to improve the goodput of the system; 3) The paper by Praveen Lalwani et al. proposed and optics inspired optimization (OIO) based cluster head selection and routing algorithm to improve the network performance.

In mobile based wireless network 1) The paper by Guangqian Xie et al. proposed an energy-efficient routing mechanism by introducing intentional mobility to wireless sensor networks (WSNs) with obstacles; 2) The paper by Chuan Zhu et al. designed a honeycomb structure based data gathering scheme, named HDGS, to optimize the data collection performance in wireless sensor networks with a mobile sink; 3) The paper by Huan Zhou et al. studied the data delivery problem in Opportunistic Mobile Networks (OppNets) and proposed a new centrality metric based on the maximum data delivery probability and a novel approach to improve the performance of data forwarding. The paper by Fu Xiao et al. addressed the data transmission schemes with opportunistic network in mobile crowd sensing and designed an energy-efficient data transmission protocol to improve the network data transmission performance.

\subsection{Performance optimization}

Lifetime is another important performance index in wireless network due to the limited energy. It is a challenging work to balance the lifetime of wireless network and other system performances. The paper by Tri Gia Nguyen et al. designed an area coverage-aware clustering protocol to efficiently use of the energy of the sensors while maintaining coverage preservation. The presented method can find an optimal number of active sensors to prolong the lifetime of the important sensors for coverage when the residual energy and the cover set are considered. The paper by Wei Wang et al. proposed a novel routing design algorithm based on variable dimension particle swarm optimization (VD-PSO) when the communication range of the nodes is taken into consideration. The paper by Yi-nan Guo et al. modeled energy-efficiency connected coverage optimization problem as a single-objective optimization problem with constraint and designed a novel evolutionary scheduling strategy to construct a full-connected network containing least sensor nodes with less energy consumption. The paper by Zhuangbin Chen et al. proposed a novel adaptation duty cycle control (ADCC) scheme based on feedback signals for WSNs, which can reduce data transmission delay to the sink node and maximize the network lifetime. The paper by Wenjun Zhang et al. formulated an infrastructure deployment and optimization (IDO) problem for Fog network, and provides an efficient heuristic algorithm named fast backward linking (FBL) to solve it.

\subsection{Protocol design}

The paper by Sushil Kumar Aanchal et al. proposed a Huffman coding and ant colony optimization based lifetime maximization (HA-LM) technique for randomly distributed WSNs. The paper by Zhijie Ma et al. proposed an optimal bandwidth allocation algorithm for heterogeneous traffic in internet of things (IoT), where both the inelastic media flow (e.g., the monitoring) and elastic data flow (e.g., the environment info from measurement) are considered. The paper by Yuan Ren et al. investigated the robust beamforming and artificial noise design is investigated in two-user interference networks with wireless information and power transfer, where the secure communication of the two pairs in the presence of an energy receiver (ER) which acts as the potential eavesdropper and intends to wiretap the confidential message transmitted to one of the information receivers (IRs) is focused. The paper by Varun Kumar Sharma et al. proposed an effective cross-layer adaptive transmission method to handle the congestion in mobile wireless ad-hoc networks by considering quick topology changes and dynamic wireless channel characteristics. In order to reduce communication packets and energy consumption, The paper by Zhen Hong et al. presented a tree-based topology construction algorithm with probability distribution and competition in the same layer (PCLT), where the PCLT calculates the weighted value of nodes through broadcasting messages and selects the best parent node using competition method in the same layer. The 
paper by Santosh Kumar Das et al. presented an intellectual energy efficient multicast routing protocol that can achieve enhanced performance over on-demand multicast routing protocol.

\subsection{Applications}

The following papers address issues of distributed control and optimization of wireless network from the viewpoint of applications. The paper by Andong Liu et al. investigated the multi mobile robots formation control problem. A distributed model predictive control (DMPC) problem was formulated and solved by Nash-optimization iteration. The paper by Zhi Li et al. considered the application of wireless network on the fire escaping. It presented a new fire spread model based on the confidential data, and designs a fast fire escaping algorithm. The paper by Zhu Ren et al. exploited wireless network to detect the important events. A multi-objective problem was formulated and a Pareto optimal solution is obtained by a general linear programming. The paper by $\mathrm{Pu}$ Yang et al. presented an optimal fault-tolerant control scheme for time-delay systems with a typical application on wireless control of the quad-rotor unmanned aerial vehicle (UAV). The paper by Dhafer Ben Arbia et al. provided a novel multi-hop bodyto-body routing protocol, namely, ORACE-Net, for public safety networks, which can enhance routing performance in disaster relief. The papers by Jiachao Chen et al. and Guangsheng Feng et al. investigated the optimization problems in cellular networks. Therein, the former provided a framework of mobile stations (MSs) energy-efficient cooperation for distributing real time content blocks (CBs), while the latter formulated the layered video transmission optimization as an unmatured probability minimization problem and solves the problem by convex optimization approach. The paper by Tianzhang Xing et al. adopted wireless networks to intelligently protect the historic relics. It provided a historical relic position monitoring system (HPMs) based on passive RFID tags.

The paper by Yourong Chen et al. presented a novel node localization algorithm where the localization accuracy of sensor nodes has been improved with the help of a mobile beacon node. The paper by Xiufang Shi et al. proposed a localization algorithm for a mobile target via hidden Markov model based channel sight condition identification, in which the Cramer-Rao Lower Bounds (CRLB) for a multi-sensor localization system under different channel sight conditions are computed analytically. The paper by Haikuo Shen et al. proposed a novel non-contact positioning measuring system where the accuracy of measurement is improved by combining a laser range finder with binocular vision sensors.
Acknowledgments In concluding this guest editorial, we would like to address our deepest gratitude to to Prof. Xuemin (Sherman) Shen, the Editor-in-Chief of Peer-to-Peer Networking and Applications for his great support and efforts throughout the whole publication process of this special issue. We are also grateful to all the authors for submitting their papers and the reviewers for their professional and timely work that helped us to select the best papers for publication. Our sincere thanks go to the editorial staff especially Ms. Melissa Fearon and Ms. Kimberly Ramos from the Springer Journal Editorial Office for their continuous support to publish this special issue.

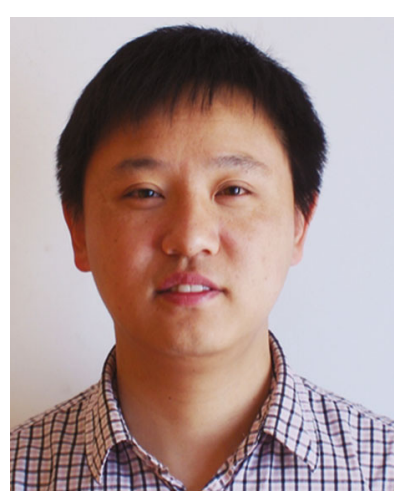

Yongmin Zhang received the $\mathrm{PhD}$ degree in control science and engineering in 2015, from Zhejiang University, Hangzhou, China. From Nov. 2013 to Jun. 2014, He was a visiting scholar with the California Institute of Technology, USA. He is currently a postdoctoral research fellow in the State Key Laboratory of Industrial Control Technology at Zhejiang University. He is a member of Networked Sensing and Control group (NESC). His research interests include Wireless Sensor Networks, resource management and Smart Grid.

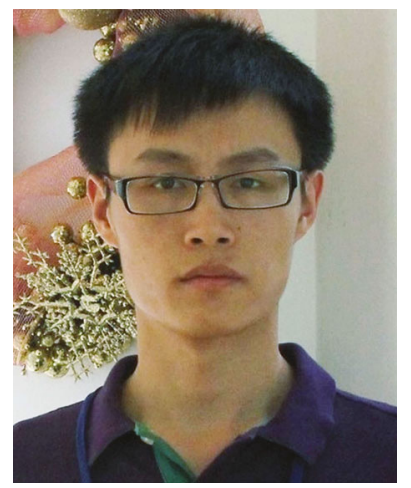

Wenchao Meng received the Ph.D. degree in control science and engineering from Zhejiang University, Hangzhou, China, in 2015. From 2013 to 2014, he was a Visiting Scholar with Arizona State University, Phoenix, AZ, USA. He is currently a Post-Doctoral Scholar with Carleton University, Ottawa, ON, Canada. His current research interests include intelligent control and smart grids.

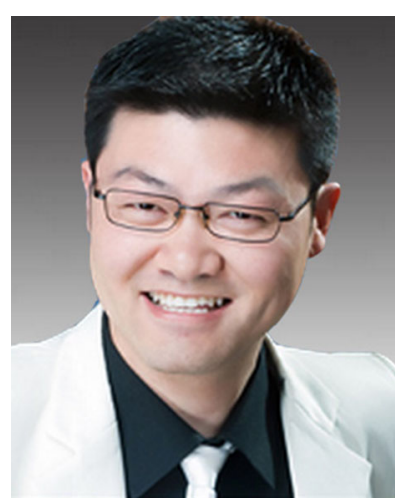

Heng Zhang received the $\mathrm{Ph} . \mathrm{D}$. degree in control science and engineering from Zhejiang University in 2015. $\mathrm{He}$ is currently an assistant professor at the School of Science, Huaihai Institute of Technology, Lianyungang, Jiangsu, China. His research interests include security and privacy in cyber-physical systems, control and optimization theory. He has been a member of IEEE and ACM member. He has served as the technical program committees of

IEEE/CIC ICCC'14, IEEE ICNC'17, and is an active reviewer of IEEE TAC, IEEE TCNS, and IEEE TIFS. 


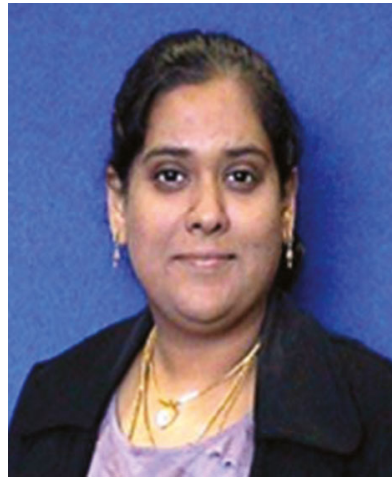

Preetha Thulasiraman is an Assistant Professor and Academic Associate for International Students in the Department of Electrical and Computer Engineering at the Naval Postgraduate School (NPS), Monterey, California, USA. She has been at NPS since January 2011. She received her $\mathrm{PhD}$ degree from the Electrical and Computer Engineering Department at the University of Waterloo, Waterloo, Ontario, Canada in September 2010, the M.Sc. degree in Computer Engineering from the University of Arizona, USA in 2006 and the B.Sc. degree in Electrical Engineering from the University of Illinois, Urbana- Champaign, USA in 2004. Since 2006 she has been involved in wireless networking research, studying various algorithmic and protocol design issues for wireless sensor, mesh and hybrid networks. Her current research interests include network layer design of resource allocation algorithms, interference management, wireless routing and fault tolerance, and general applications of graph theory. She is currently pursuing interests in unmanned ad hoc wireless networks and Internet of Things networks with an emphasis on efficient routing, communication and security protocols, mobility management and performance evaluation. She has been a member of IEEE and ACM member since 2006. She has served on the technical program committees of several IEEE and ACM sponsored conferences. She has contributed to over 26 research papers in the area of wireless networks.

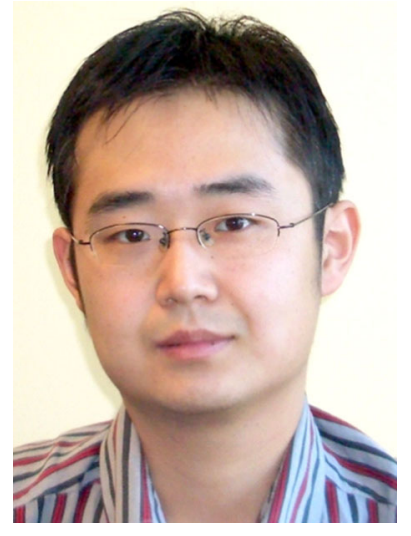

Tom H. Luan received the B.Sc. degree from Xian Jiaotong University, China, in 2004, the M.Phil. degree from Hong Kong University of Science and Technology in 2007, and Ph.D. degrees from the University of Waterloo in 2012. Since December 2013, he has been the Lecturer in Mobile and Apps at the School of Information Technology, Deakin University, Melbourne Burwood, Australia. His research mainly focuses on vehicular networking, wireless content distribution, peer-to-peer networking and mobile cloud computing. 